\title{
Finite dipole model for extreme near-field thermal radiation between a tip and planar $\mathrm{SiC}$ substrate
}

\author{
Amun Jarzembski, Keunhan Park \\ Department of Mechanical Engineering, University of Utah, Salt Lake City, UT 84112, \\ USA
}

\begin{abstract}
Recent experimental studies have measured the infrared (IR) spectrum of tip-scattered near-field thermal radiation for a $\mathrm{SiC}$ substrate and observed up to a $50 \mathrm{~cm}^{-1}$ redshift of the surface phonon polariton ( $\mathrm{SPhP}$ ) resonance peak $[1,2]$. However, the observed spectral redshift cannot be explained by the conventional near-field thermal radiation model based on the point dipole approximation. In the present work, a heated tip is modeled as randomly fluctuating point charges (or fluctuating finite dipoles) aligned along the primary axis of a prolate spheroid, and quasistatic tip-substrate charge interactions are considered to formulate the effective polarizability and self-interaction Green's function. The finite dipole model (FDM), combined with fluctuational electrodynamics, allows the computation of tip-plane thermal radiation in the extreme near-field (i.e., $H / R \lesssim 1$, where $H$ is the tip-substrate gap distance and $R$ is the tip radius), which cannot be calculated with the point dipole approximation. The FDM provides the underlying physics on the spectral redshift of tip-scattered near-field thermal radiation as observed in experiments. In addition, the $\mathrm{SPhP}$ peak in the near-field thermal radiation spectrum may split into two peaks as the gap distance decreases into the extreme near-field regime. This observation suggests that scatteringtype spectroscopic measurements may not convey the full spectral features of tip-plane extreme near-field thermal radiation.
\end{abstract}

Keywords: Near-field thermal radiation, Thermal infrared near-field spectroscopy, Finite dipole model, Tip-sample interactions, Surface phonon polaritons

Preprint submitted to Journal of Quantitative Spectroscopy and Radiative TransferJanuary 4, 2017

(C) 2016. This manuscript version is made available under the Elsevier user license

http://www.elsevier.com/open-access/userlicense/1.0/ 


\section{Introduction}

Thermal radiation in the near-field is very different from the far-field phenomenon. Previous theoretical studies have predicted that in the near-field, or when the emitter-receiver separation is less than the thermal wavelength, thermal radiation can exceed Planck's blackbody limit by up to several orders of magnitude due to radiation tunneling of evanescent electromagnetic (EM) waves [3-6], and that its spectrum becomes quasi-monochromatic if surface modes are excited $[5,7,8]$. Recently, significant efforts have been made to experimentally validate the near-field enhancement of thermal radiation for plane-plane [9-15] and sphere-plane [16-19] configurations. However, in these configurations, achieving sub-10 nm vacuum gap distances is challenging due to technical difficulties in precision emitter-receiver gap control. The smallest vacuum gap distances achieved to date for the plane-plane and sphere-plane geometry are $60 \mathrm{~nm}$ and $20 \mathrm{~nm}$, respectively [14, 19].

In efforts to explore extreme near-field thermal radiation within sub$10 \mathrm{~nm}$ gap distances, scanning probe microscopy has been used due to its capability of controlling the tip position with sub-nanometer resolution. To measure near-field radiative heat transfer from a heated substrate to a tip, Kittel's group used a thermocouple-integrated needle probe to measure the tip temperature for different tip-substrate gap distances down to $1 \mathrm{~nm}$ in a ultra-high vacuum scanning tunneling microscope [20]. A similar experiment was conducted by measuring the near-field thermal conductance between a scanning thermal microscope probe and suspended microheater device [21]. However, their results are not consistent with each other, leaving open questions about the extreme near-field behaviors of thermal radiation. Tip-based near-field optical measurement is another scheme to investigate extreme nearfield thermal radiation. De Wilde et al. [22] imaged the electromagnetic local density of states by collecting tip-scattered thermal emission from a metallic tip scanning over a heated substrate. Recently, tip-scattered near-field thermal radiation between a tip and $\mathrm{SiC}$ substrate was spectroscopically analyzed to demonstrate that thermal emission can excite surface phonon polaritons (SPhPs) on a $\mathrm{SiC}$ substrate to emit quasi-monochromatic thermal radiation $[1,2]$. However, they observed a spectral redshift of the $\mathrm{SPhP}$ resonance peak up to $50 \mathrm{~cm}^{-1}$, which could not be explained with the conventional point dipole model $[1,2]$. This trait of scattering-type thermal infrared nearfield spectroscopy (TINS) necessitates the development of a comprehensive model to understand the underlying physics of extreme near-field thermal 
radiation in the tip-plane geometry.

The point dipole model (PDM) is a conventional model that reduces the complex tip geometry to a spherical point dipole located at the tip end [7, 2325]. Figure 1(a) illustrates the schematic of the PDM, where $R$ represents the tip (or dipole) radius and $H$ is the tip-substrate separation equivalently defined throughout the paper. Quasistatic tip-substrate interactions are derived by mirroring the entire dipole in the surface and developing recursive interactions between the tip and image dipoles. The mathematical formulation of the PDM has been well developed for different tip-substrate configurations [7, 24-26], and it has been used to understand the tip-substrate near-field interactions in tip-based optical nanospectroscopy and near-field thermal radiation experiments $[1,20,27,28]$. However, the PDM is only valid for tip-substrate separations much larger than the tip radius to satisfy the uniform electric field condition of the model [24]. This limitation leads to a discrepancy between the PDM and the measured near-field IR spectrum for polar materials [1, 28-31]. For example, Babuty et al. [1] had to use the tip geometry of $R=1.6 \mu \mathrm{m}$ and $H=100 \mathrm{~nm}$ to replicate the observed spectral redshift of tip-scattered thermal radiation with the PDM, which is an unrealistic tip geometry outside the valid regime of the PDM. Aside from the simple PDM, various numerical methods have been developed to compute near-field thermal radiation between arbitrary geometries, such as the finite difference time domain method [32, 33], molecular dynamics [34], and thermal discrete dipole approximation $[35,36]$. However, these numerical schemes are computationally expensive to be routinely used in conjunction with experiments.

In this article, we implement the finite dipole model (FDM) to calculate tip-plane near-field thermal radiation for tip-plane separations less than those permitted by the PDM while maintaining computational simplicity. In section 2, we provide a detailed description of the PDM to evaluate its limitations, followed by the FDM and its adaptation with fluctuational electrodynamics to formulate the effective polarizability and self-interaction Green's function. Section 3 presents and discusses the results obtained by the FDM, comparing them with both the PDM and experimentally obtained spectra from the literature. 


\section{Modeling}

Near-field radiative heat transfer between a tip and a planar surface can be modeled by treating the tip as a randomly fluctuating electric dipole, $\mathbf{p}_{t}$. Based on fluctuational electrodynamics, the net spectral radiative heat transfer from the dipole at $T_{t}$ to a planar surface at $T_{s}$ or vice versa can be written as $[23,24,37,38]$

$$
P_{\mathrm{net}}^{t \rightarrow s}(\omega)=\frac{2}{\pi}\left[\Theta\left(\omega, T_{t}\right)-\Theta\left(\omega, T_{s}\right)\right] \times \operatorname{Tr}\left[\operatorname{Im}\left(\stackrel{\leftrightarrow}{\alpha}_{\mathrm{eff}}\right) \operatorname{Im}\left[\stackrel{\leftrightarrow}{\mathbf{G}}_{R}\left(\mathbf{r}_{t}, \mathbf{r}_{t}\right)\right]\right]
$$

where $\Theta(\omega, T)$ is the mean energy of a Planck oscillator at the angular frequency $\omega$ in thermal equilibrium at temperature $T$ and is given as $\Theta(\omega)=$ $\hbar \omega /\left[\exp \left(\hbar \omega / k_{B} T\right)-1\right]$, where $\hbar$ is the reduced Planck constant and $k_{B}$ is the Boltzmann constant. The effective electric polarizability tensor is denoted by $\stackrel{\leftrightarrow}{\alpha}_{\text {eff }}$, and $\stackrel{\leftrightarrow}{\mathbf{G}}_{R}\left(\mathbf{r}_{t}, \mathbf{r}_{t}\right)$ is the dyadic reflection Green's function for the electric field at the dipole position, $\mathbf{r}_{t}$, referred to as the self-interaction Green's function throughout this paper. Eq. (1) clearly indicates that the effective polarizability of the dipole representing the tip and the self-interaction Green's function are the key variables for tip-plane near-field thermal radiation.

For a point dipole in free space subjugated to the unperturbed, uniform electric field, $\mathbf{E}_{o}$, the dipole moment is solely induced by $\mathbf{E}_{o}$ to formulate $\mathbf{p}_{o}=\stackrel{\leftrightarrow}{\alpha}_{o}^{P D} \mathbf{E}_{o}$, where $\stackrel{\leftrightarrow}{\alpha}_{o}^{P D}$ is the bare electric polarizability tensor of the point dipole. For the special case of a spherical point dipole as shown in Fig. 1(a), $\stackrel{\leftrightarrow}{\alpha}_{o}^{P D}$ is given by $[7]$

$$
\stackrel{\leftrightarrow}{\alpha}_{o}^{P D}=4 \pi R^{3}\left(\frac{\varepsilon_{t}-1}{\varepsilon_{t}+2}\right) \stackrel{\leftrightarrow}{\mathbf{I}}
$$

where $\varepsilon_{t}$ is the tip's dielectric function and $\stackrel{\leftrightarrow}{\mathbf{I}}$ is the unit tensor. When the point dipole approaches a surface, the dipole moment is induced by the total electric field to yield $\mathbf{p}_{t}=\stackrel{\leftrightarrow}{\alpha}_{o}^{P D} \mathbf{E}_{\text {tot }}\left(\mathbf{r}_{t}\right)$, where $\mathbf{E}_{\text {tot }}$ includes the reflection of the electric field radiated from the dipole itself. The total electric field at the dipole position, $\mathbf{r}_{t}$, is thus written as [24]

$$
\mathbf{E}_{\mathrm{tot}}\left(\mathbf{r}_{t}\right)=\mathbf{E}_{o}+\stackrel{\leftrightarrow}{\mathbf{G}}_{R}\left(\mathbf{r}_{t}, \mathbf{r}_{t}\right) \mathbf{p}_{t}
$$

where $\stackrel{\leftrightarrow}{\mathbf{G}}_{R}\left(\mathbf{r}_{t}, \mathbf{r}_{t}\right)$ is the self-interaction Green's function and for a point dipole 
can be approximated as $[24,39]$

$$
\stackrel{\leftrightarrow}{\mathbf{G}}_{R}^{P D}\left(\mathbf{r}_{t}, \mathbf{r}_{t}\right)=\frac{\beta}{32(H+R)^{3} \pi \varepsilon_{o}}\left[\begin{array}{lll}
1 & 0 & 0 \\
0 & 1 & 0 \\
0 & 0 & 2
\end{array}\right]
$$

using the image dipole method. Here, $\varepsilon_{o}$ is the vacuum permittivity, and $\beta$ is the quasistatic Fresnel reflection coefficient for $p$-polarization written as $\beta=\left(\varepsilon_{s}-1\right) /\left(\varepsilon_{s}+1\right)$, where $\varepsilon_{s}$ is the dielectric function of the substrate. The effective electric polarizability that accounts for the near-field interaction between a point dipole and a planar surface can be derived from Eq. (3) $[1,24]$ :

$$
\stackrel{\leftrightarrow}{\alpha}_{\mathrm{eff}}^{P D}=\frac{\stackrel{\leftrightarrow}{\alpha}_{o}^{P D}}{\stackrel{\leftrightarrow}{\mathbf{I}}-\stackrel{\leftrightarrow}{\alpha}_{o}^{P D} \stackrel{\leftrightarrow}{\mathbf{G}}_{R}^{P D}\left(\mathbf{r}_{t}, \mathbf{r}_{t}\right)}
$$

The total dipole moment $\mathbf{p}_{t}$ can thus be expressed with $\stackrel{\leftrightarrow}{\alpha}$ eff to yield $\mathbf{p}_{t}=$ $\stackrel{\leftrightarrow}{\alpha}_{\text {eff }}^{P D} \mathbf{E}_{o}$

As illustrated in Figs. 1(b) and (c), the FDM simplifies a conductive tip to a vertically aligned prolate spheroid to better represent the field enhancement due to the antenna-like nature of the actual tip geometry within the quasistatic approximation $\left(2 L_{p}<\lambda\right)$ [29]. In fact, the FDM has shown good correlations with the experimental near-field optical spectra of polar materials [29-31]. Due to the antenna-like tip shape, the electric field parallel to the tip axis (or $p$-polarized) should play a dominant role over the $s$-polarized electric field in tip-plane near-field radiative heat transfer. The net radiative heat transfer rate can thus be simplified to the scalar form:

$$
P_{\text {net }}^{t \rightarrow s}(\omega)=\frac{2}{\pi}\left[\Theta\left(\omega, T_{t}\right)-\Theta\left(\omega, T_{s}\right)\right] \times\left[\operatorname{Im}\left(\alpha_{\mathrm{eff}, z z}\right) \operatorname{Im}\left[G_{R, z z}\left(\mathbf{r}_{t}, \mathbf{r}_{t}\right)\right]\right]
$$

where only the $z z$-components of $\stackrel{\leftrightarrow}{\alpha}_{\text {eff }}$ and $\stackrel{\leftrightarrow}{\mathbf{G}}_{R}\left(\mathbf{r}_{t}, \mathbf{r}_{t}\right)$ in Eq. (1) are taken into account: see Appendix Appendix A for further discussion. When the tip is placed in free space, the FDM represents the tip with two point charges (monopoles), $Q_{o}$ and $-Q_{o}$, located on the primary axis of the spheroid and a distance $W_{o}$ away from each end. This point charge distribution provides the best approxamation of the scattered electric field in the near vicinity of a conducting prolate spheroid located in a uniform $p$-polarized external electric field $\mathbf{E}_{o}[29]$. The $z z$-component of the bare polarizability tensor due to $Q_{o}$ 
and $-Q_{o}$ can be expressed as $\alpha_{o, z z}^{F D}=p_{o, z} / E_{o, z}$ to formulate [29]

$$
\alpha_{o, z z}^{F D}=2 w L_{p}^{3}\left(\gamma_{o}-1\right)
$$

where $w$ is the location of $Q_{o}$ normalized by the spheroid semi-major axis given by $W_{o} / L_{p}$, and $\gamma_{o}$ represents the analytic electric field enhancement at the spheroid apex and can be expressed as [40]

$$
\gamma_{o}=1+\frac{\frac{2}{r}+\ln \left(\frac{0.5 r}{2-0.5 r}\right)}{2(1-0.5 r)\left(\frac{1 / r-\varepsilon_{t}}{\varepsilon_{t}-1}\right)-\ln \left(\frac{0.5 r}{2-0.5 r}\right)},
$$

where $r$ is the normalized tip radius given by $r=R / L_{p}$.

When the spheroid tip is placed in the near-field of a planar surface, strong near-field interactions between $Q_{o}$ and its mirroring monopole $Q_{o}^{\prime}$ should alter the charge distribution in the spheroid. As discussed in Appendix B, a point charge in vicinity of the conducting spheroid induces a line charge distribution inside the spheroid, having a sharp peak at the focal point and oscillation along the major axis. In the FDM, this charge distribution was approximated with induced monopoles $Q_{i}$ and $-Q_{i}$, located at the spheroid focal point and at the spheroid center, respectively [29]. It should be noted that the induced monopoles are solely due to the near-field interactions of the lower $Q_{o}$ with the substrate, not the entire dipole as addressed by the PDM. The presence of the induced monopoles $\left(Q_{i}\right.$ and $\left.-Q_{i}\right)$ adds another finite dipole $\mathbf{p}_{i}$ to formulate the total finite dipole as $\mathbf{p}_{t}=\mathbf{p}_{o}+\mathbf{p}_{i}$. Therefore, the FDM-based effective polarizability that includes the tip-substrate near-field interaction can be written as $[29,30]$

$$
\alpha_{\mathrm{eff}, z z}^{F D}=\alpha_{o, z z}^{F D}(1+\eta),
$$

where $\eta$ is the near-field contrast factor, defined as

$$
\eta=\frac{p_{i, z}}{p_{o, z}}=\frac{\beta(2 g-2 h-w-r) \ln (h+w / 2+r / 4)}{4 \ln (r / 4)-\beta(4 g-4 h-3 r) \ln (h+r / 2)},
$$

which represents the relative strength of the near-field interaction. Here, $h=H / L_{p}$ and $g$ quantifies the amount of charge induced in $Q_{i}$ due to single reflection from the substrate. The derivation of $g$ can be found in Appendix Appendix B. The equivalent self-interaction Green's function under the FDM 
scheme can be determined from Eq. (3). Since the total finite dipole moment and the total electric field (both $z$-components) can be expressed as $p_{t, z}=$ $\alpha_{\text {eff }, z z}^{F D} E_{o, z}$ and $E_{\text {tot }, z}=E_{o, z}(1+\eta)$ from Eq. (9), respectively, substituting these relations into Eq. (3) yields the FDM self-interaction Green's function:

$$
G_{R, z z}^{F D}\left(\mathbf{r}_{t}, \mathbf{r}_{t}\right)=\frac{\eta}{\alpha_{\mathrm{eff}, z z}^{F D}}
$$

It should be noted that the derived self-interaction Green's function is consistent with Eq. (5) in terms of its inverse proportionality to the effective polarizability and its dependence on the tip geometry, tip-substrate separation, and the optical properties of both the tip and the substrate.

For the tip-plane configuration, the direct measurement of the spectral distribution of near-field thermal radiation is challenging. Instead, near-field thermal radiation scattered by the tip has been measured and spectroscopically analyzed as an indirect spectral measurement scheme [1, 2, 41, 42]. The time-averaged Poynting vector of tip-scattered electromagnetic field at the detector can be approximated as [1, 24]

$$
\left\langle S_{d}\right\rangle=\frac{\varepsilon_{o} c_{0}}{2}\left|\left(\stackrel{\leftrightarrow}{g}+\stackrel{\leftrightarrow}{g}_{R}\right) \stackrel{\leftrightarrow}{\alpha}_{\mathrm{eff}} \mathbf{E}_{o}\right|^{2} r_{d}^{2} d \Omega
$$

where $c_{0}$ is the speed of light in free space, $\stackrel{\leftrightarrow}{g}$ and $\stackrel{\leftrightarrow}{g}_{R}$ are the far-field direct and reflected Green's functions, respectively, $r_{d}$ is the tip-detector distance, and $d \Omega$ is the solid angle subtended by the detector [39]. The quadratic expression of $\mathbf{E}_{0}$ in Eq. (12) represents the electric component of the electrodynamic local density of states at the tip position and is proportional to the imaginary component of the self-interaction Green's function [1, 23]. Therefore, the tipscattered radiative power at the detector can be expressed as

$$
\left\langle S_{d}\right\rangle \propto \operatorname{Tr}\left\{\left|\left(\stackrel{\leftrightarrow}{g}+\stackrel{\leftrightarrow}{g}_{R}\right) \stackrel{\leftrightarrow}{\alpha}_{\text {eff }}\right|^{2} \operatorname{Im}\left[\stackrel{\leftrightarrow}{\mathbf{G}}_{R}\left(\mathbf{r}_{t}, \mathbf{r}_{t}\right)\right]\right\}
$$

\section{Results and Discussion}

To critically evaluate the FDM formulations, Fig. 2 shows the imaginary component of the effective polarizability, $\alpha_{\text {eff }, z z}$, normalized with the dipole's volume and the real and imaginary components of the self-interactions Green's function, $G_{R, z z}\left(\mathbf{r}_{t}, \mathbf{r}_{t}\right)$, for both the PDM and FDM at different tip-substrate separations. Both models consider a gold tip with $R=20 \mathrm{~nm}$ in the near-field of a planar SiC substrate. The Drude model [43] and the Lorentz oscillator 
model [7] were used to determine the dielectric functions of gold and $\mathrm{SiC}$, respectively. Figure 2 clearly shows that both models exhibit the resonance behavior of $\mathrm{SiC}$ in the effective polarizability and self-interaction Green's function. However, as shown in Figs. 2(a), (b), and (c), the PDM does not shift the resonance peak position from $948 \mathrm{~cm}^{-1}$, which is intrinsically the $\mathrm{SPhP}$ mode of $\mathrm{SiC}$. Although $\operatorname{Im}\left[\alpha_{\mathrm{eff}, z z}\right]$ shows a small redshift of $3 \mathrm{~cm}^{-1}$ at $H=10 \mathrm{~nm}$ (or $H / R=0.5$ ) in Fig. 2(a), it is not large enough to explain the redshift observed in experiments $[1,2]$. On the other hand, The FDM, as shown in Figs. 2(d), (e), and (f), predicts a significant redshift of the resonance peak in both $\alpha_{\mathrm{eff}, z z}$ and $G_{R, z z}\left(\mathbf{r}_{t}, \mathbf{r}_{t}\right)$ as the tip-substrate separation decreases. In the FDM, the presence of the tip modifies the local dispersion relation of $\mathrm{SiC}$ to shift the $\mathrm{SPhP}$ resonance to lower frequencies. Although $\alpha_{\mathrm{eff}, z z}$ and $G_{R, z z}\left(\mathbf{r}_{t}, \mathbf{r}_{t}\right)$ are coupled as expressed in Eq. (11), $\alpha_{\mathrm{eff}, z z}$ represents the near-field interaction as experienced by the tip dipole while $G_{R, z z}\left(\mathbf{r}_{t}, \mathbf{r}_{t}\right)$ represents the EM field near the tip due to reflection from the surface. Therefore, multiple reflections of EM waves between the tip and the substrate should enhance the dipole moment of the tip, which in turn affects the EM field around the tip. It should be noted that since $\alpha_{\mathrm{eff}, z z}$ describes the tendency of the tip to form a charge distribution, its change due to near-field interactions can propagate into the far-field through scattering. However, the information within $G_{R, z z}\left(\mathbf{r}_{t}, \mathbf{r}_{t}\right)$ is confined near the surface and quickly decays as the EM wave is scattered into the far-field. In addition, Figs. 2(d) and (f) show that $\alpha_{\mathrm{eff}, z z}$ experiences a larger redshift than $G_{R, z z}\left(\mathbf{r}_{t}, \mathbf{r}_{t}\right)$ as the gap distance decreases, which will affect the spectral distribution of near-field thermal radiation.

Figure 3 compares the near-field spectral power densities emitted from a gold tip with $20 \mathrm{~nm}$ in radius to a planar $\mathrm{SiC}$ substrate, computed by the PDM and FDM for different tip-substrate separations. For better comparison, the near-field radiative power density was calculated by dividing Eq. (6) with the volume of the model's dipole (i.e., spherical dipole for the PDM and spheroidal dipole for the FDM). The PDM in Fig. 3(a) does not show any redshift or splitting of the $\mathrm{SiC} \mathrm{SPhP}$ resonance because the effective polarizability and self-interaction Green's function do not change the peak position over the range of $H$ studied. The PDM's incapability to predict the spectral redshift is the main reason for the discrepancy between measured and computed tip-plane near-field thermal radiation spectra. When using the FDM, however, the near-field radiative power density experiences a redshift as the tip-substrate gap decreases and exhibits two distinct peaks at $H=10 \mathrm{~nm}$ (or 
$H / R=0.5)$ as shown in Fig. 3(b). Here the more redshifted peak is associated with the effective polarizability and the less shifted peak is due to the self-interaction Green's function. The spectral difference between $\alpha_{\mathrm{eff}, z z}$ and $G_{R, z z}\left(\mathbf{r}_{t}, \mathbf{r}_{t}\right)$ in the FDM leads to the split of the SPhP resonance at small $H$, which has been similarly observed in the optical force gradient spectrum on a dipole in the vicinity of a resonant sample surface [44]. Figure 3(c) shows the tip-scattered intensity at a far-field detector as modeled by the FDM, which predicts a single resonance with the spectral redshift. As shown in Fig. 2, $\alpha_{\mathrm{eff}, z z}$ is more sensitive to near-field interactions than $G_{R, z z}\left(\mathbf{r}_{t}, \mathbf{r}_{t}\right)$. When near-field thermal radiation is scattered to the far-field, the gap-dependence of $\alpha_{\text {eff }, z z}$ is radiated through the far-field Green's functions, $\stackrel{\leftrightarrow}{g}$ and $\stackrel{\leftrightarrow}{g}_{R}$, while the contribution of $G_{R, z z}\left(\mathbf{r}_{t}, \mathbf{r}_{t}\right)$ to the near-field thermal radiation spectrum does not propagate as strongly [1,23].

In Fig. 4, we compare the FDM result of tip-scattered near-field thermal radiation with thermal infrared near-field spectroscopy (TINS) measurement data for a heated silicon tip scanning over a $\mathrm{SiC}$ substrate [2]. To model the silicon tip, the dielectric function of silicon was taken from Ref. [45] and $g$ was set to 0.8 reflecting the charge distribution induced in the semiconductor tip $[2,29]$. The FDM was fit to the experimental data for three spectral shifts observed: (a) $5 \mathrm{~cm}^{-1}$, (b) $25 \mathrm{~cm}^{-1}$, and (c) $50 \mathrm{~cm}^{-1}$. In each case, the tipsubstrate separation was assumed to be constant at $5 \mathrm{~nm}$ which leaves the tip radius, $R$, and probe length, $L_{p}$, as the remaining fitting parameters. To account for the experimental spectrometer resolution, the FDM results have been convoluted with spectral resolutions of (a) $46 \mathrm{~cm}^{-1}$, (b) $16 \mathrm{~cm}^{-1}$, and (c) $50 \mathrm{~cm}^{-1}$, which are in good agreement with the resolutions provided by Ref. [2] $\left(49 \mathrm{~cm}^{-1}, 11 \mathrm{~cm}^{-1}\right.$, and $39 \mathrm{~cm}^{-1}$, respectively). As expected, the TINS spectra does not show the resonance splitting due to its scattering measurement scheme, and is in good agreement with the FDM results for tipscattered thermal radiation. The smallest redshift of $5 \mathrm{~cm}^{-1}$ occurs because of relatively weak near-field interactions from a sharp and short tip $(R=5 \mathrm{~nm}$ and $L_{p}=16.7 \mathrm{~nm}$ ). To model the $50 \mathrm{~cm}^{-1}$ redshift, the tip radius was increased to $30 \mathrm{~nm}$, and the probe length was increased to $3 \mu \mathrm{m}$. For the $25 \mathrm{~cm}^{-1}$ redshift, the tip radius was $12 \mathrm{~nm}$, and the probe length was $120 \mathrm{~nm}$. The obtained trend of fitting parameters indicates that a long probe with a dull tip may cause a larger redshift in the tip-scattered thermal infrared near-field spectrum of $\mathrm{SiC}$.

The effects of tip geometry on the tip-scattered thermal infrared nearfield spectrum are further studied by calculating the spectral redshift for 
different tip radii, probe lengths and tip-substrate gap distances when a gold tip and a SiC substrate are considered: See Fig. 5. For better comparison, the tip aspect ratio is defined as $L_{p} / R$ and is used instead of $L_{p}$ in the figure. The grey-hatched lines indicate regions that are not computationally supported by the FDM, which will be explained in more details in Fig. 6 . In Fig. 5(a), the spectral redshift increases as the tip-substrate separation $(H)$ decreases and the tip radius $(R)$ increases. Conceptually, a bigger tip radius and a smaller gap distance enhance multiple reflections between the tip and substrate to alter the spectrum of the tip dipole moment. The linear proportionality between $H$ and $R$ for constant redshift contours suggests that when a dull tip is used for experiments, the tip-substrate gap distance should be increased to reduce the spectral redshift of the measured spectrum. On the other hand, as shown in Fig. 5(b), the spectral redshift increases as the tip aspect ratio increases. For longer probes, the tip dipole moment becomes stronger due to the increase of separation between charges in the tip. However, the effect of the probe length is not as prominent for a sharper tip (i.e., small $R$ ). It is thus imperative to use a sharp tip to suppress the spectral redshift in the tip-scattered thermal infrared near-field spectrum.

Figure 6 compares the total power density predicted by the FDM and PDM over a large range of tip-substrate separations for a $20 \mathrm{~nm}$ radius gold tip and planar $\mathrm{SiC}$ substrate. The PDM predicts that near-field thermal radiation monotomically increases with a $1 / d^{3}$ gap dependence until it is saturated at $H / R \approx 1$. However, the validity of the PDM is restricted to $H / R \gg 1$, otherwise the electric field cannot be considered uniform across the dipole [24]. Joulain et al. [24] suggested a lower limit of $H / R \sim 2$; however, no rigorous justification has been provided. On the other hand, the minimum gap distance of the FDM is determined by the valid regime of fluctuational electrodynamics. Previous studies have revealed that fluctuational electrodynamics may not be sufficient to describe near-field thermal interactions if the separation gap becomes of the order or smaller than the lattice constant of a material [46], for which nonlocal effects of the dielectric function [47, 48] as well as quantum electronic coupling effects [49] should be considered. The upper limit of the FDM is $H / R \approx 4$, above which the induced charge inside the tip becomes more uniformly distributed and thus cannot be approximated with a point charge, $Q_{i}$. However, the near-field radiative power density calculated with the FDM converges to the PDM result at $H / R \approx 4$, demonstrating consistency between the FDM formulations and the widely accepted PDM. Furthermore, based on the FDM analysis a more 
practical lower limit of the PDM is $H / R \sim 4$ where the two models converge. At $H / R<4$, the FDM predicts quicker saturation of the power density than the PDM, approaching a value around two orders of magnitude smaller than the PDM prediction. We believe that the PDM's over-prediction of the nearfield power density is due to the simplification of the tip as a spherical point dipole subjected to strong EM fields near the surface. However, the FDM may predict a larger near-field heat transfer rate because the volume of the finite dipole is typically a couple of orders of magnitude larger than that of the point dipole.

\section{Conclusions}

In this article, we have implemented the FDM as a simple dipole-based model for the calculation of thermal radiation in the extreme near-field regime. When the tip-substrate separation is smaller than the tip radius, the FDM predicts the spectral redshift of the surface phonon polariton resonance peak up to $50 \mathrm{~cm}^{-1}$, as observed in experiments. The underlying physics of such redshift is due to the strong near-field interactions between charges in the tip apex and near the surface of the substrate. Therefore, the amount of redshift is sensitive to the tip geometry (i.e., tip radius and tip length) and the tip-substrate separation. The FDM also predicts that the thermal radiation spectrum in the extreme near-field may have resonance splitting due to different near-field effects on the polarizability and the electromagnetic field distribution, which may not be fully revealed by tip-scattered thermal infrared near-field spectroscopy. The obtained results will allow for better interpretation and design of future tip-plane near-field thermal radiation experiments.

\section{Acknowledgments}

This work was supported by the National Science Foundation (CBET1403072 and CBET-1605584) and startup support at the University of Utah. AJ also acknowledges financial supports from the University of Utah's Sid Green Fellowship and the National Science Foundation Graduate Research Fellowship (No. 2016213209). The authors thank Prof. Mathieu Francoeur at the University of Utah for his valuable inputs. 


\section{References}

[1] A. Babuty, K. Joulain, P.-O. Chapuis, J.-J. Greffet, Y. De Wilde, Blackbody Spectrum Revisited in the Near Field, Phys. Rev. Lett. 110 (2013) 146103.

[2] B. T. O'Callahan, W. E. Lewis, A. C. Jones, M. B. Raschke, Spectral frustration and spatial coherence in thermal near-field spectroscopy, Phys. Rev. B 89 (2014) 245446.

[3] D. Polder, M. Van Hove, Theory of radiative heat transfer between closely spaced bodies, Phys. Rev. B 4 (1971) 3303-3314.

[4] J. Loomis, H. Maris, Theory of heat transfer by evanescent electromagnetic waves, Phys. Rev. B 50 (1994) 18517-18524. doi:10.1103/PhysRevB.50.18517.

[5] S. Basu, Z. M. Zhang, C. Fu, Review of Near-Field Thermal Radiation and Its Application to Energy Conversion, Int. J. of Energ. Res. 33 (2009) 1203-1232.

[6] K. Park, Z. M. Zhang, Fundamentals and Applications of Near-Field Radiative Energy Transfer, Front. Heat Mass Transf. 4 (2013) 013001.

[7] J.-P. Mulet, K. Joulain, R. Carminati, J.-J. Greffet, Nanoscale Radiative Heat Transfer Between a Small Particle and a Plane Surface, Appl. Phys. Lett. 78 (2001) 2931-2933.

[8] M. Francoeur, S. Basu, S. J. Petersen, Electric and magnetic surface polariton mediated near-field radiative heat transfer between metamaterials made of silicon carbide particles., Opt. Express 19 (2011) 18774-88.

[9] L. Hu, A. Narayanaswamy, X. Chen, G. Chen, Near-Field Thermal Radiation Between Two Closely Spaced Glass Plates Exceeding Planck's Blackbody Radiation Law, Appl. Phys. Lett. 92 (2008) 133106.

[10] R. S. Ottens, V. Quetschke, S. Wise, A. A. Alemi, R. Lundock, G. Mueller, D. H. Reitze, D. B. Tanner, B. F. Whiting, Near-Field Radiative Heat Transfer between Macroscopic Planar Surfaces, Phys. Rev. Lett. 107 (2011) 014301. 
[11] R. St-Gelais, B. Guha, L. Zhu, S. Fan, M. Lipson, Demonstration of strong near-field radiative heat transfer between integrated nanostructures, Nano Lett. 14 (2014) 6971-6975.

[12] M. Lim, S. S. Lee, B. J. Lee, Near-field thermal radiation between doped silicon plates at nanoscale gaps, Phys. Rev. B 91 (2015) 195136.

[13] K. Ito, A. Miura, H. Iizuka, H. Toshiyoshi, Parallel-plate submicron gap formed by micromachined low-density pillars for near-field radiative heat transfer, Appl. Phys. Lett. 106 (2015) 083504.

[14] B. Song, D. Thompson, A. Fiorino, Y. Ganjeh, P. Reddy, E. Meyhofer, Radiative heat conductances between dielectric and metallic parallel plates with nanoscale gaps, Nat. Nanotechnol. 11 (2016) 509-514.

[15] R. St-Gelais, L. Zhu, S. Fan, M. Lipson, Near-field radiative heat transfer between nanostructures in the deep sub-wavelength regime, Nat. Nanotechnol. 11 (2016) 515-519.

[16] A. Narayanaswamy, S. Shen, G. Chen, Near-Field Radiative Heat Transfer Between a Sphere and a Substrate, Phys. Rev. B 78 (2008) 115303.

[17] E. Rousseau, A. Siria, G. Jourdan, S. Volz, F. Comin, J. Chevrier, J.J. Greffet, Radiative heat transfer at the nanoscale, Nat. Photonics 3 (2009) 514-517.

[18] S. Shen, A. Narayanaswamy, G. Chen, Surface phonon polaritons mediated energy transfer between nanoscale gaps, Nano Lett. 9 (2009) 29092913.

[19] B. Song, Y. Ganjeh, S. Sadat, D. Thompson, A. Fiorino, V. FernándezHurtado, J. Feist, F. J. Garcia-Vidal, J. C. Cuevas, P. Reddy, E. Meyhofer, Enhancement of near-field radiative heat transfer using polar dielectric thin films, Nat. Nanotechnol. 10 (2015) 253-258.

[20] A. Kittel, W. Muller-Hirsch, J. Parisi, S. A. Biehs, D. Reddig, M. Holthaus, Near-Field Heat Transfer in a Scanning Thermal Microscope, Phys. Rev. Lett. 95 (2005) 224301.

[21] K. Kim, B. Song, V. Fernández-Hurtado, W. Lee, W. Jeong, L. Cui, D. Thompson, J. Feist, M. T. H. Reid, F. J. García-Vidal, J. C. Cuevas, 
E. Meyhofer, P. Reddy, Radiative heat transfer in the extreme near field, Nature 528 (2015) 387-391.

[22] Y. De Wilde, F. Formanek, R. Carminati, B. Gralak, P.-A. Lemoine, K. Joulain, J.-P. Mulet, Y. Chen, J.-J. Greffet, Thermal radiation scanning tunnelling microscopy., Nature 444 (2006) 740-3. doi:10.1038/nature05265.

[23] K. Joulain, J.-P. Mulet, F. Marquier, R. Carminati, J.-J. Greffet, Surface electromagnetic waves thermally excited: Radiative heat transfer, coherence properties and Casimir forces revisited in the near field, Surf. Sci. Rep. 57 (2005) 59-112.

[24] K. Joulain, P. Ben-Abdallah, P.-O. Chapuis, Y. De Wilde, a. Babuty, C. Henkel, Strong tip-sample coupling in thermal radiation scanning tunneling microscopy, J. Quant. Spectrosc. Radiat. Transfer 136 (2014) $1-15$.

[25] J. Aizpurua, T. Taubner, F. J. García de Abajo, M. Brehm, R. Hillenbrand, Substrate-enhanced infrared near-field spectroscopy, Opt. Express 16 (2008) 1529.

[26] O. Huth, F. Rüting, S.-A. Biehs, M. Holthaus, Shape-dependence of near-field heat transfer between a spheroidal nanoparticle and a flat surface, Eur. Phys. J. Appl. Phys. 50 (2010) 10603.

[27] R. Hillenbrand, T. Taubner, F. Keilmann, Phonon-enhanced light matter interaction at the nanometre scale., Nature 418 (2002) 159-62.

[28] T. Taubner, F. Keilmann, R. Hillenbrand, Nanomechanical resonance tuning and phase effects in optical near-field interaction, Nano Lett. 4 (2004) 1669-1672.

[29] N. Ocelic, Quantitative Near-field Phonon-Polariton Spectroscopy, Ph.D. thesis, Max Planck Institute (2007).

[30] A. Cvitkovic, N. Ocelic, R. Hillenbrand, Analytical model for quantitative prediction of material contrasts in scattering-type near-field optical microscopy., Opt. Express 15 (2007) 8550-65. 
[31] S. Amarie, F. Keilmann, Broadband-infrared assessment of phonon resonance in scattering-type near-field microscopy, Phys. Rev. B 83 (2011) 045404 .

[32] A. W. Rodriguez, O. Ilic, P. Bermel, I. Celanovic, J. D. Joannopoulos, M. Soljačić, S. G. Johnson, Frequency-Selective Near-Field Radiative Heat Transfer between Photonic Crystal Slabs: A Computational Approach for Arbitrary Geometries and Materials, Phys. Rev. Lett. 107 (2011) 114302.

[33] K. H. A. Datas, D. Hirashima, FDTD Simulation of Near-Field Radiative Heat Transfer between Thin Films Supporting Surface Phonon Polaritons: Lessons Learned, J. Therm. Sci. Tech. 8 (2013) 91-105.

[34] G. Domingues, S. Volz, K. Joulain, J.-J. Greffet, Heat Transfer Between Two Nanoparticles Through Near Field Interaction, Phys. Rev. Lett. 94 (2005) 85901.

[35] S. Edalatpour, M. Francoeur, The Thermal Discrete Dipole Approximation (T-DDA) for near-field radiative heat transfer simulations in threedimensional arbitrary geometries, J. Quant. Spectrosc. Radiat. Transfer 133 (2014) 364-373.

[36] S. Edalatpour, M. Francoeur, Near-field radiative heat transfer between arbitrarily-shaped objects and a surface, Phys. Rev. B 94 (2016) 045406.

[37] S. Rytov, Y. Kravtsov, V. Tatarskii, Principles of Statistical Radiophysics, Vol. 3, Springer, New York, 1989.

[38] S. J. Petersen, S. Basu, M. Francoeur, Near-field thermal emission from metamaterials, Photonics Nanostruct. 11 (2013) 167-181.

[39] L. Novotny, B. Hecht, Principles of Nano-Optics, Cambridge University Press, Cambridge, 2006.

[40] N. Calander, M. Willander, Theory of surface-plasmon resonance optical-field enhancement at prolate spheroids, J. Appl. Phys. 92 (2002) 4878-4884.

[41] A. C. Jones, M. B. Raschke, Thermal infrared near-field spectroscopy, Nano Lett. 12 (2012) 1475-81. 
[42] A. C. Jones, B. T. O'Callahan, H. U. Yang, M. B. Raschke, The thermal near-field: Coherence, spectroscopy, heat-transfer, and optical forces, Prog. Surf. Sci. 88 (2013) 349-392.

[43] M. A. Ordal, R. J. Bell, J. R. W. Alexander, L. L. Long, M. R. Querry, Optical properties of fourteen metals in the infrared and far infrared: $\mathrm{Al}, \mathrm{Co}, \mathrm{Cu}, \mathrm{Au}, \mathrm{Fe}, \mathrm{Pb}, \mathrm{Mo}, \mathrm{Ni}, \mathrm{Pd}, \mathrm{Pt}, \mathrm{Ag}, \mathrm{Ti}, \mathrm{V}$, and W, Appl. Opt. 24 (1985) 4493-4499.

[44] H. U. Yang, M. B. Raschke, Resonant optical gradient force interaction for nano-imaging and -spectroscopy, New J. Phys. 18 (2016) 053042.

[45] E. D. Palik, Handbook of optical constants of solids, Vol. 3, Academic Press, San Diego, CA, 1998.

[46] Y. Ezzahri, K. Joulain, Vacuum-induced phonon transfer between two solid dielectric materials: Illustrating the case of Casimir force coupling, Phys. Rev. B 90 (2014) 115433.

[47] C. Henkel, K. Joulain, Electromagnetic field correlations near a surface with a nonlocal optical response, Appl. Phys. B 84 (2006) 61-68.

[48] F. Singer, Y. Ezzahri, K. Joulain, Near field radiative heat transfer between two nonlocal dielectrics, J. Quant. Spectrosc. Radiat. Transfer 154 (2015) 55-62.

[49] V. Chiloyan, J. Garg, K. Esfarjani, G. Chen, Phonon heat conduction at sub-nanometre gaps, Nat. Commun. 6 (2015) 1-7.

[50] I. V. Lindell, G. Dassios, K. I. Nikoskinen, Electrostatic image theory for the conducting prolate spheroid, J. Phys. D: Appl. Phys. 34 (2001) 2302-2307. 


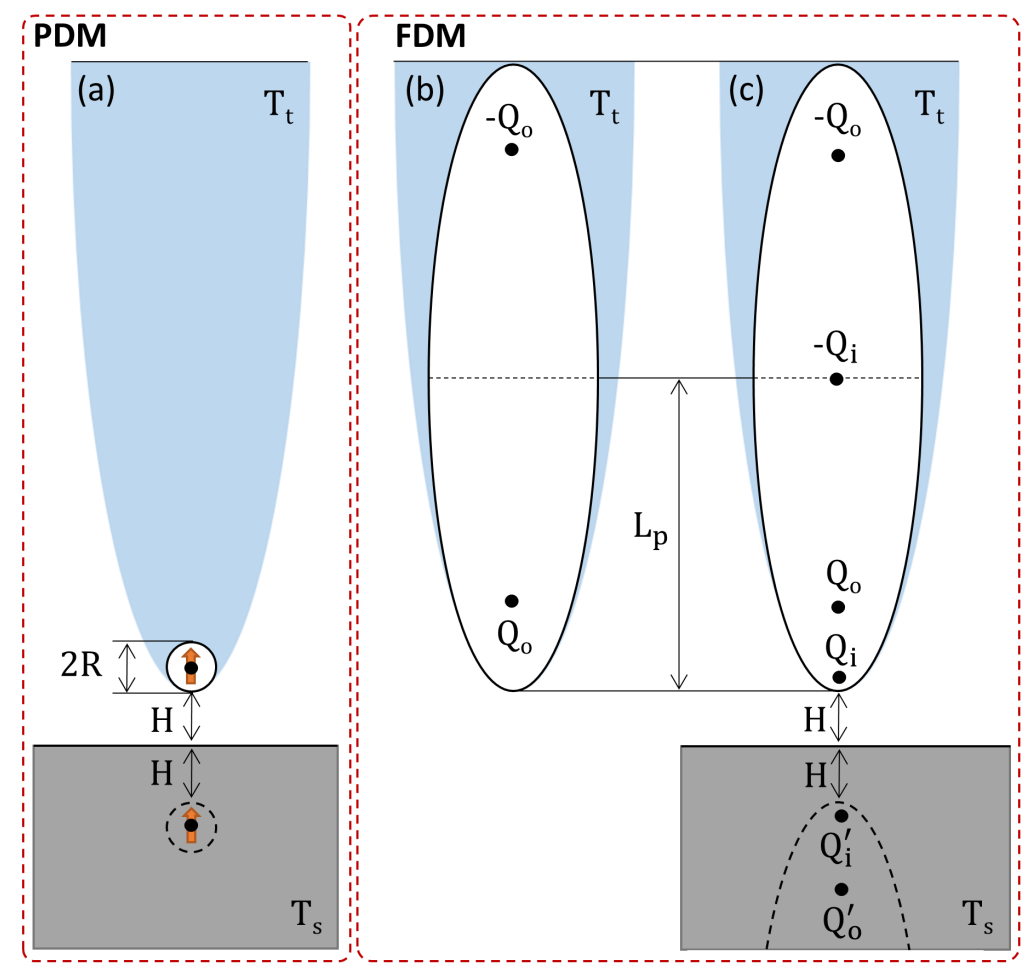

Figure 1: Schematic of a tip with radius $R$ and temperature $T_{t}$ a distance $H$ above a surface at temperature $T_{s}$ as modeled by (a) the PDM, (b) the FDM with no surface, and (c) the FDM near the surface. The FDM uses a prolate spheroidal geometry with semi-major axis $L_{p}$. The non-perturbed charge distributions, $Q_{o}$ and $-Q_{o}$, are formed by incident electric field without the surface while the induced charge distributions, $Q_{i}$ and $-Q_{i}$, are induced by the near-field interaction between the tip and surface quantified by $Q_{o}^{\prime}$ and $Q_{i}^{\prime}$. 

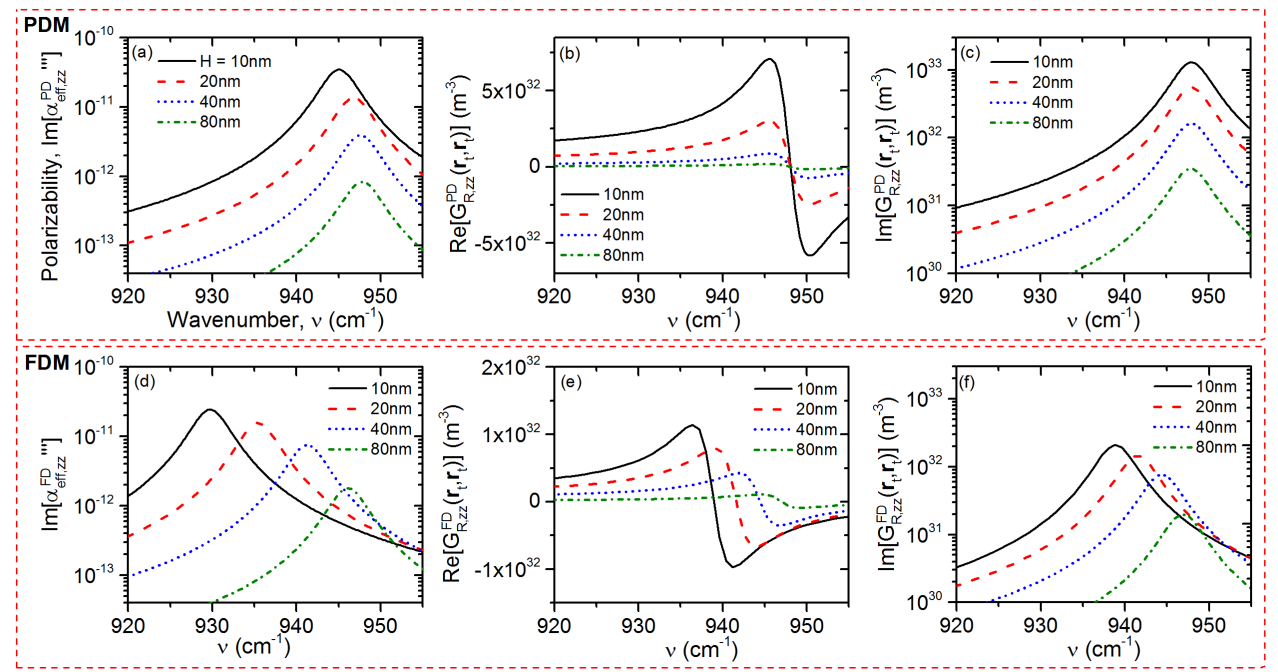

Figure 2: The $z$-component of the effective polarizability, normalized with the model's dipole volume, for a $20 \mathrm{~nm}$ radius gold tip over a $\mathrm{SiC}$ substrate modeled with (a) PDM and (d) FDM or the tip aspect ratio of $L_{p} / R=10$. The real and imaginary parts of the $z$-component of the self-interaction Green's function are also shown in $(\mathrm{b}, \mathrm{c})$ for the PDM and in (e,f) for the FDM, respectively. While the PDM shows little to no spectral redshift below the tip-substrate separation of $H=4 R$, the FDM predicts the systematic redshift in both the effective polarizability and the self-interaction Green's function although their shifted amounts are different at the same tip-substrate separation. 


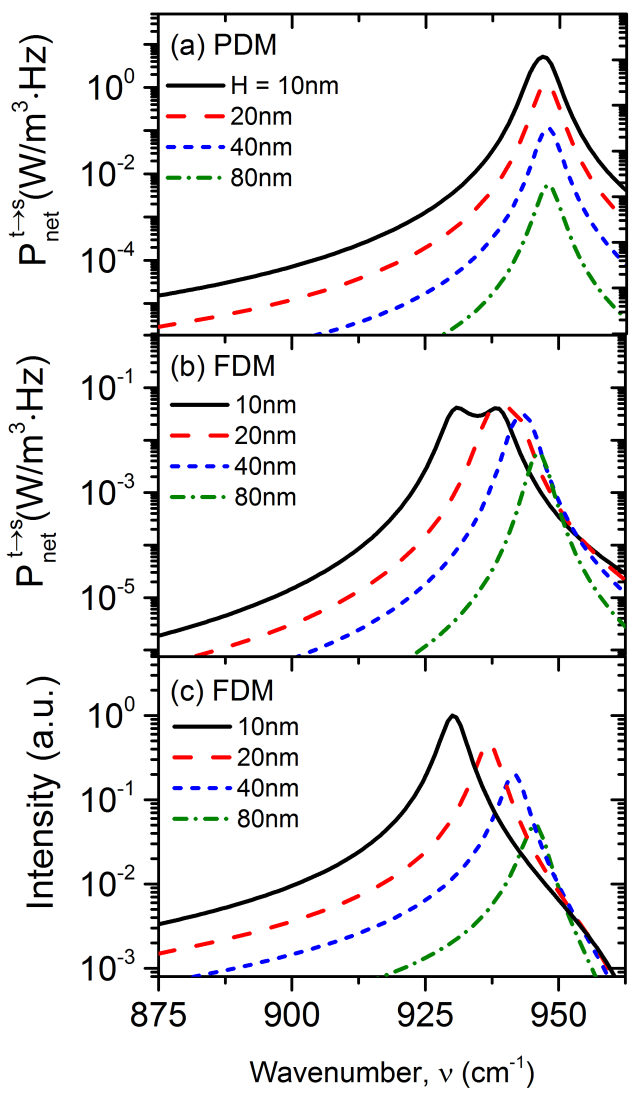

Figure 3: Spectral near-field radiative power density from a $20 \mathrm{~nm}$ gold tip at $300 \mathrm{~K}$ to a SiC substrate at $0 \mathrm{~K}$, calculated with (a) the PDM and (b) the FDM; and (c) tip-scattered near-field thermal infrared spectrum calculated using the FDM for $L_{p} / R=10$. For tipsubstrate separations less than $H=4 R$, the FDM predicts the spectral redshift of the $\mathrm{SiC}$ SPhP resonance as well as its splitting into two identifiable peaks at $H=10 \mathrm{~nm}$ (or $H / R=0.5)$. However, the tip-scattered near-field thermal infrared spectrum does not convey the resonance splitting at $H=10 \mathrm{~nm}$. 


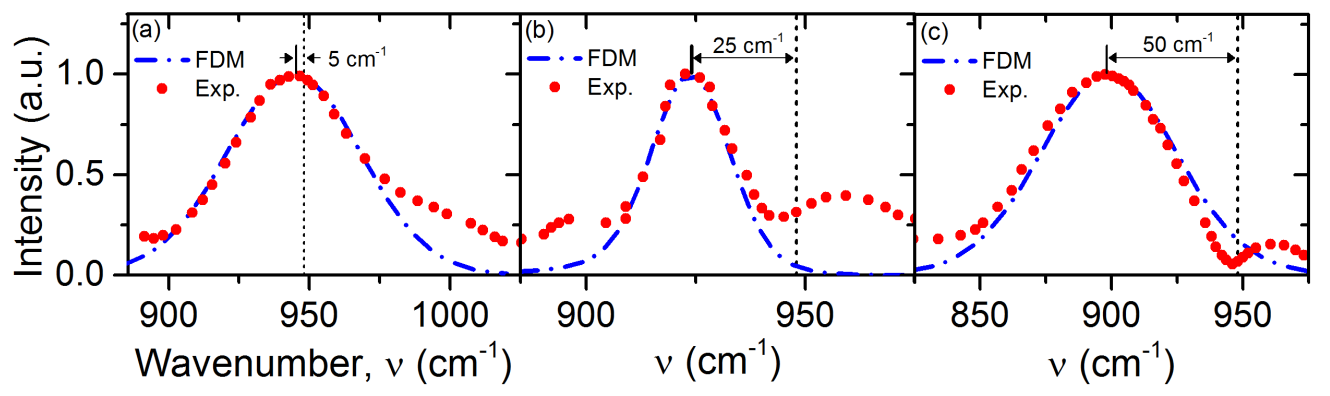

Figure 4: Comparison between the FDM and experimental TINS results from Ref. [2]. The FDM was calculated for a fixed tip-substrate separation of $5 \mathrm{~nm}$ and $g=0.8$. Both results are in excellent agreement for spectral redshifts of (a) $5 \mathrm{~cm}^{-1}$ with fitting parameters: $R=5 \mathrm{~nm} \& L_{p}=16.7 \mathrm{~nm}$, (b) $25 \mathrm{~cm}^{-1}$ with fitting parameters: $R=12 \mathrm{~nm} \& L_{p}=$ $120 \mathrm{~nm}$, and (c) $50 \mathrm{~cm}^{-1}$ with fitting parameters: $R=30 \mathrm{~nm} \& L_{p}=3 \mu \mathrm{m}$. 


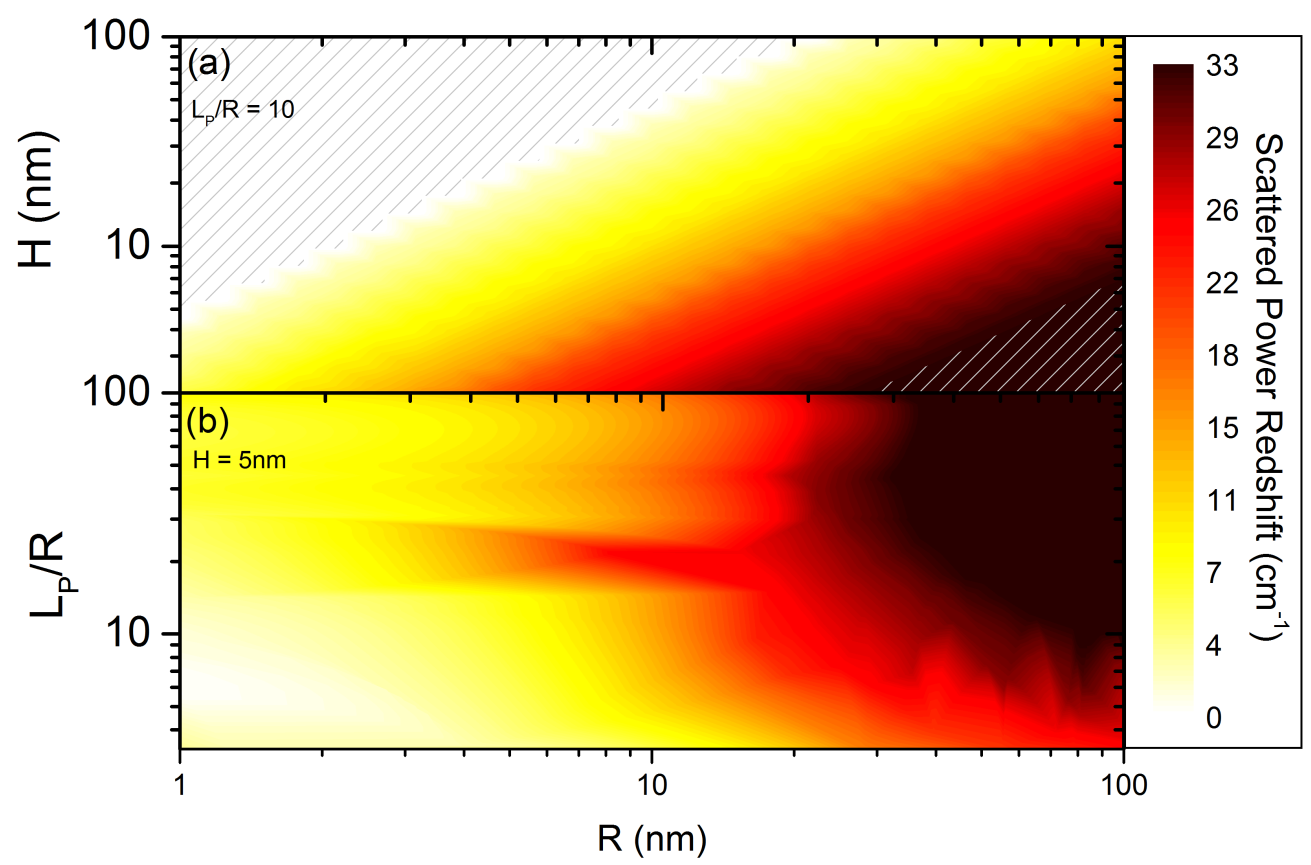

Figure 5: Spectral redshift contours in the tip-scattered near-field thermal radiation spectrum, depending on tip radius, $R$, and (a) tip-substrate separation, $H$, or (b) tip aspect ratio, $L_{p} / R$, as determined for a gold tip over $\mathrm{SiC}$ substrate. The spectral redshift increases for a larger tip radius and tip aspect ratio, but it decreases for larger tip-substrate separations. 


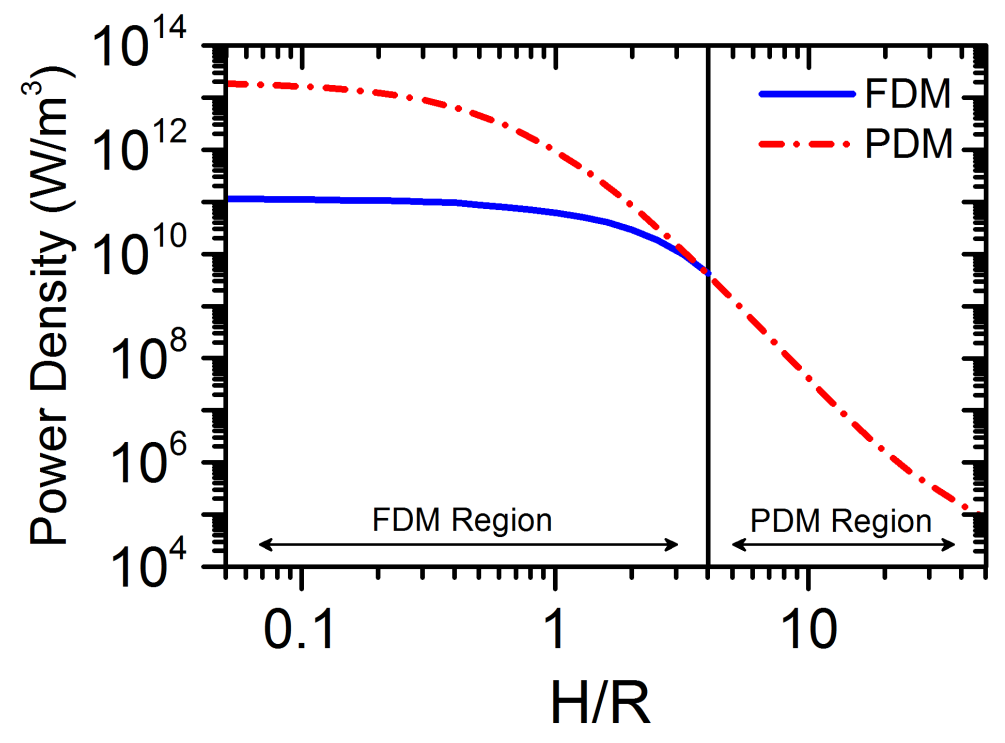

Figure 6: Comparison of total near-field radiative power densities calculated by the PDM and the FDM for a $20 \mathrm{~nm}$ gold tip at $300 \mathrm{~K}$ and a $\mathrm{SiC}$ substrate at $0 \mathrm{~K}$. The FDM used the tip aspect ratio of $L_{p} / R=10$ for calculation. In the region where the PDM is not valid (i.e. $H<4 R$ ), the FDM saturates the power density more quickly to become approximately two orders of magnitude smaller than the PDM predication. However, the FDM converges to the PDM at $H=4 R$, demonstrating consistency between the derivations of both models. 


\section{Appendix A. Scalar assumption of the near-field thermal radiation power equation}

For scattering-type near-field scanning optical microscopy, the dipole moments oriented perpendicularly to the tip axis can be neglected, as the incident electric field is generally polarized parallel to the tip axis to maximize its antenna effect. However, for thermal radiation, the polarization state of the EM field is essentially random due to the excitation of both $x, y$ and $z$-oriented dipoles. In this section, use of Eq. (6) is justified by evaluating the general PDM for prolate spheroidal dipoles when horizontal dipoles (i.e., dipole moments oriented perpendicularly to the tip axis) are neglected. In general, the bare electric dipole polarizability for a prolate spheroidal point dipole is an anisotropic tensor given by the diagonal components [26]

$$
\alpha_{P, i i}=V_{E}\left[\frac{\varepsilon_{t}-1}{1+n_{i}\left(\varepsilon_{t}-1\right)}\right] \quad(i=x, y, z)
$$

Here, $V_{E}$ is the dipole volume, $\varepsilon_{t}$ is the dielectric function of the tip, and $n_{i}$ is a depolarization coefficient defined for a prolate spheroid as [26]

$$
\begin{gathered}
n_{x}=n_{y}=0.5\left(1-n_{z}\right), \\
n_{z}= \begin{cases}\frac{1-\xi^{2}}{2 \xi^{3}}\left[\ln \left(\frac{1+\xi}{1-\xi}\right)-2 \xi\right], & L_{s}<L_{p} \\
\frac{1+\xi^{2}}{\xi^{3}}\left[\xi-\tan ^{-1}(\xi)\right], & L_{s}>L_{p},\end{cases}
\end{gathered}
$$

where $L_{s}$ and $L_{p}$ are the semi-minor and semi-major axes of the prolate spheroid, respectively, and $\xi$ is the ellipticity of the prolate spheroid defined as

$$
\xi^{2}=\left|1-\frac{L_{s}^{2}}{L_{p}^{2}}\right|
$$

Equations (4) and (5) are the general forms of the effective point-dipole polarizability and self-interaction Green's function, respectively, and are applicable for a prolate spheroidal point dipole with bare polarizability given by Eq. (A.1).

Figure A.7 compares the total near-field power calculated using Eqs. (1) and (6) for a prolate spheroid point dipole as a function of the tip aspect ratio $L_{p} / R$. These calculations were performed for a gold tip with a radius of $R=$ 
$20 \mathrm{~nm}$ over a SiC substrate with a tip-substrate separation of $H=100 \mathrm{~nm}$ to satisfy the PDM's inherent assumptions. The percent difference is less than $2.5 \%$ as the tip aspect ratio decreases from 100 to 10 and is still less than $10 \%$ at $L_{p} / R=3.3$, which is the smallest tip aspect ratio considered in the present study. This small deviation illustrates that the $x, y$-components of the net radiative heat transfer rate vector are negligible in comparison to the dominant $z$-component. While an equivalent calculation has not been shown for the FDM, one can expect less contribution from the $x, y$-components due to the further enhancement of the z-component predicted by the FDM. 


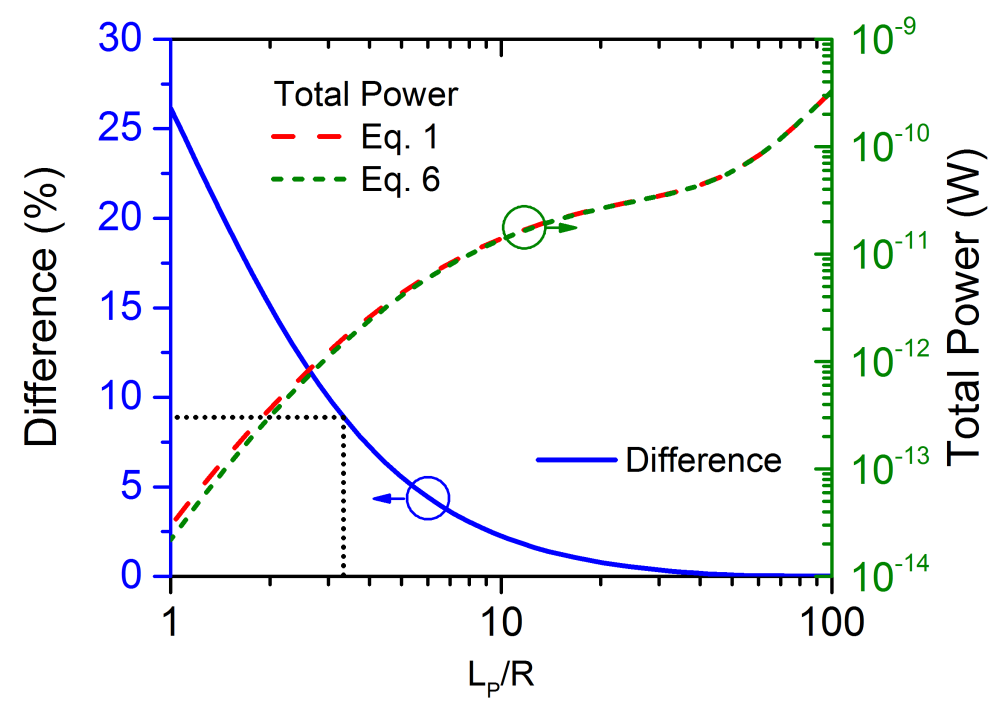

Figure A.7: Numerical deviations between Eqs. (1) and (6) as a function of the tip aspect ratio when the tip is modeled as a spheroidal point dipole. The comparison illustrates less than $10 \%$ deviation for all tip tip aspect ratios used in this article (i.e. $L_{p} / R>3.33$ ) and even less than $2.5 \%$ for $L_{p} / R>10$, demonstrating that the $z$-component of the near-field radiative power is dominant over the $x, y$-components. 


\section{Appendix B. Calculation of the induced point charge in a conduct- ing tip due to substrate}

The quantity $g$ represents the amount of charge induced in the tip through a single reflection from the sample surface, and is a central quantity needed to formulate the near-field contrast factor, $\eta$. The formulation of $g$ begins with the distribution of charge induced in a conductive spheroid due to an external point charge, $Q_{e}$, located at $D$ from the center of the spheroid: see Fig. B.8(a). The induced charge distribution along the tip axes can be expressed as [50]

$q_{i}\left(z^{\prime}\right)=-\frac{Q_{e}}{2 F} \sum_{m=0}^{m_{\max }}(2 m+1) \frac{\mathscr{L}_{m}(D / F) \mathscr{P}_{m}\left(L_{p} / F\right)}{\mathscr{L}_{m}\left(L_{p} / F\right)} \mathscr{P}_{m}\left(z^{\prime} / F\right) \times \mathrm{U}\left[F^{2}-\left(z^{\prime}\right)^{2}\right]$,

where $z^{\prime}$ is measured from the spheroid center (positive axis towards tip apex), $F$ is the geometric focal point of the prolate spheroid, $D$ is the distance to $Q_{e}$ measured from the spheroid center, $\mathscr{L}_{m}$ is the associated Legendre Function of the second-kind $m$-th order, $\mathscr{P}_{m}$ is the Legendre Polynomial $m$ th order, and U is the Heaviside unit step function. However, Eq. (B.1) does not converge well for $D_{a}<1.5 R$, where $D_{a}=D-L_{p}$. For this condition, the induced charge distribution is approximated with a point charge at location, $h_{\mathrm{c}}$, and a continuous line charge along the spheroid axis [50]:

$$
q_{i}\left(z^{\prime}\right)=q_{i, \mathrm{c}}\left(z^{\prime}\right)+q_{i, l}\left(z^{\prime}\right),
$$

where

$$
q_{i, \mathrm{c}}\left(z^{\prime}\right)=-Q_{e} \sqrt[4]{\frac{h_{\mathrm{c}}^{2}-F^{2}}{D^{2}-F^{2}}} \delta\left(z^{\prime}-h_{\mathrm{c}}\right)
$$

and

$$
\begin{aligned}
q_{i, l}\left(z^{\prime}\right)=-\frac{Q_{e}}{2 F} & \sum_{m=0}^{m_{\max }}(2 m+1)\left(\frac{\mathscr{L}_{m}(D / F) \mathscr{P}_{m}\left(L_{p} / F\right)}{\mathscr{L}_{m}\left(L_{p} / F\right)}\right. \\
& \left.-\sqrt[4]{\frac{h_{\mathrm{c}}^{2}-F^{2}}{D^{2}-F^{2}}} \mathscr{P}_{m}\left(h_{\mathrm{c}} / F\right)\right) \mathscr{P}_{m}\left(z^{\prime} / F\right) \times \mathrm{U}\left[F^{2}-\left(z^{\prime}\right)^{2}\right] .
\end{aligned}
$$

The location of the point charge, $h_{\mathrm{c}}$, is determined by

$$
h_{\mathrm{c}}=\frac{L_{p}^{2}+L_{s}^{2}}{F^{2}} D-2 \frac{L_{s} L_{p}}{F^{2}} \sqrt{D^{2}-F^{2}} .
$$


In Eqs. (B.1) - (B.4), $m_{\max }$ represents the amount of terms considered in the series summations and is assumed to be 9 for ideal convergence. Use of excess terms in the Legendre function series summations results in immediate divergence [50].

The induced charge distribution in a conductive spheroid $q_{i}\left(z^{\prime}\right)$ due to point charge $Q_{e}$ is shown in Fig. B.8(b) and (c) for $D_{a}=1 R$ and $D_{a}=2 R$, respectively. In both cases, a large portion of the induced charge resides at the spheroid focal point, while the remainder oscillates about the spheroid center. Since performing recursive interactions between the line charge distribution in the tip and the sample is quite formidable, $q_{i}\left(z^{\prime}\right)$ is simplified to a point charge at the spheroid focus by assuming that the charge distribution near the tip apex, i.e., $L_{p}-\left(R+D_{a}\right)<z^{\prime}<L_{p}$, is the primary contributor to the near-field interaction. The amount of the induced point charge due to the external charge, $Q_{e}$, can be expressed with a normalized factor $g$, defined as

$$
g=\frac{\int_{L_{p}-\left(R+D_{a}\right)}^{L_{p}} q_{i, l}\left(z^{\prime}\right) d z^{\prime}+q_{i, \mathrm{c}}\left(h_{\mathrm{c}}\right)}{\int_{-L_{p}}^{L_{p}} q_{i, t}\left(z^{\prime}\right) d z^{\prime}},
$$

where the numerator represents the amount of the induced point charge by integrating $q_{i}\left(z^{\prime}\right)$ near the tip apex. Once $g$ is determined as a function of $D_{a}$, the amount of the total induced point charge $Q_{i}$ can be calculated recursively by considering the mirror charges in the substrate, $Q_{i}^{\prime}$ and $Q_{0}^{\prime}$ : see Fig. 1(c). However, it should be noted that $g$ is derived based on the perfectly conductive spheroid assumption, and the adjustment of $g$ is required to satisfy charge neutrality on the surface. To this end, we assume that the opposite charge $-Q_{i}$ is uniformly distributed across the spheroid. Therefore, $g$ should be adjusted to $g-\left(R+D_{a}\right) / 2 L_{p}$, where $\left(R+D_{a}\right) / 2 L_{p}$ represents the fraction of $-Q_{i}$ that exists within $R+D_{a}$ of the tip apex $[29,30]$. In the literature, the constant value of $g$ has been assumed approximately 0.7 for conductive tips and 0.8 for silicon tips [29-31]. However, we directly calculated $g$ using Eq. (B.1) to Eq. (B.6) for better accuracy in this article. 


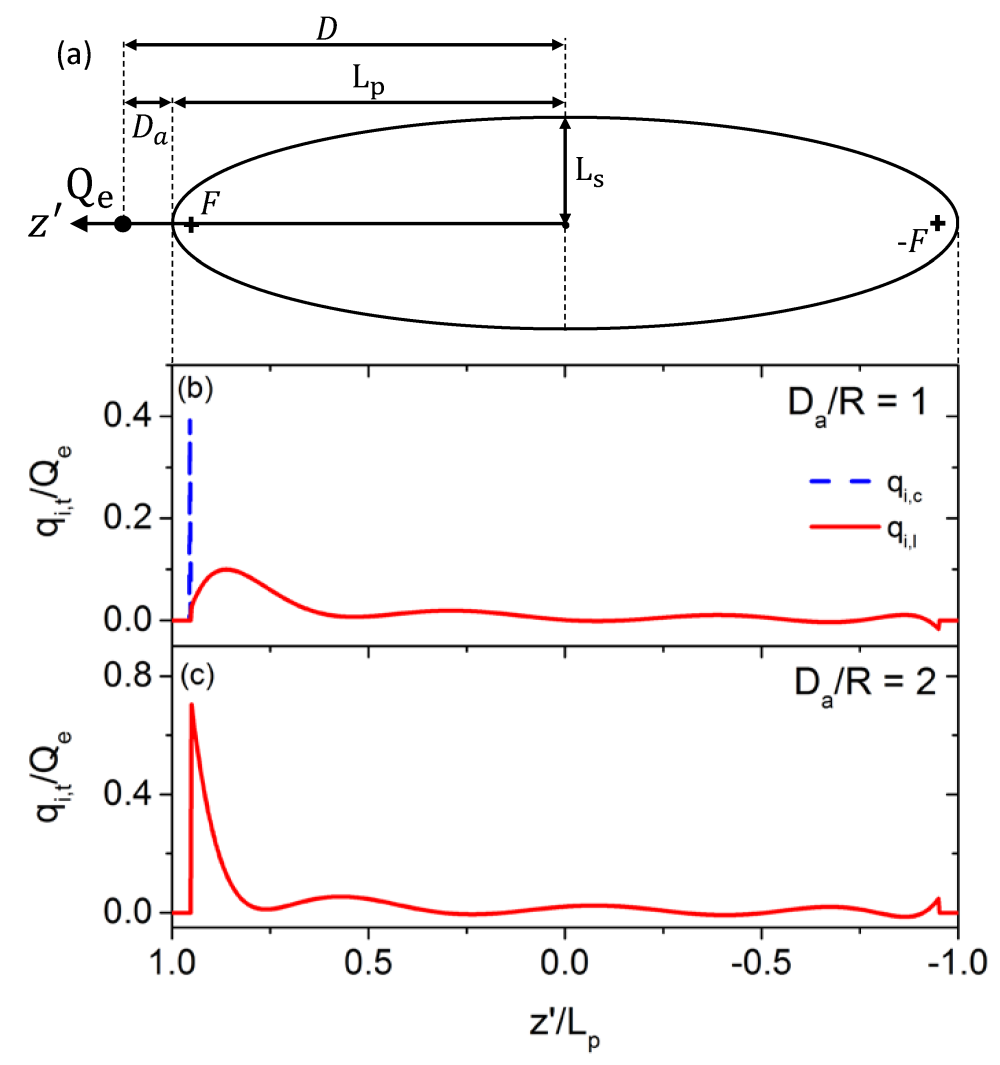

Figure B.8: (a) Geometry used to determine the line charge distribution induced in a conductive spheroid due to the external point charge $Q_{e}$ located $D_{a}$ from the spheroid apex (or $D$ from the spheroid center) and the calculated charge distributions induced by $Q_{e}$ located at (b) $z^{\prime}=R+L_{p}$ using Eqs. (B.2) through (B.5) and (c) $z^{\prime}=2 R+L_{p}$ using Eq. (B.1). 